\title{
Efficacy and safety of humanized CD19 CAR-T as a salvage therapy for recurrent CNSL of B-ALL following murine CD19 CAR-T cell therapy
}

\author{
XIN LI ${ }^{1}$, MEI-JING LIU ${ }^{1,2}$, NAN MOU ${ }^{3}$, ZHEN-XING YANG ${ }^{3}$, \\ JIA WANG ${ }^{1}$, JUAN MU ${ }^{1}$, HAI-BO ZHU ${ }^{1}$ and QI DENG ${ }^{1}$ \\ ${ }^{1}$ Department of Hematology, Tianjin First Central Hospital, Tianjin 300192; \\ ${ }^{2}$ Department of Hematology, The First Central Clinical College of Tianjin Medical University, \\ Tianjin 300192; ${ }^{3}$ Shanghai Genbase Biotechnology Co., Ltd., Tianjin 201210, P.R. China
}

Received July 8, 2019; Accepted February 19, 2021

DOI: 10.3892/ol.2021.13049

\begin{abstract}
The present study aimed to compare the differences between the humanized CD19 chimeric antigen receptor (CAR)-T cell therapy and the murine CD19 CAR-T therapy in recurrent B-acute lymphoblastic leukemia (B-ALL). A 62-year-old male patient who had B-ALL $\left(\mathrm{BCR} / \mathrm{ABL}^{+}\right)$ for 4 years was diagnosed with relapsed central nervous system leukemia (CNSL). After several courses of high dose methotrexate combined with intrathecal chemotherapy, the patient received murine CD19 CAR-T therapy and achieved complete response (CR). The patient was diagnosed with relapsed CNSL again 15 months after his murine CD19 CAR-T therapy, and was therefore enrolled in the humanized CD19 CAR-T therapy. Subsequently, the present study aimed to compare murine and humanized CD19 CAR-T cells against Nalm- 6 cells in vitro and in mice. The patient initially achieved CR from his murine CD19 CAR-T therapy with Grade 1 cytokine-release syndrome (CRS) and Grade 1 CAR-T cell-related encephalopathy syndrome (CRES). The patient then achieved CR again from his humanized CD19 CAR-T therapy with Grade 1 CRS and Grade 2 CRES. Peak levels of CD19 CAR-T cells were higher in humanized CD19 CAR-T therapy than those in murine CD19 CAR-T therapy 7 days after infusion in the peripheral blood, in bone marrow and in cerebrospinal fluid (CSF). The cytokine levels were higher in humanized CD19 CAR-T therapy than those in murine CD19 CAR-T therapy in the peripheral blood and in CSF. The cytotoxicity to Nalm-6 cells was higher in humanized CD19 CAR-T cells than that in murine CD19 CAR-T cells in vitro.
\end{abstract}

Correspondence to: Dr Qi Deng, Department of Hematology, Tianjin First Central Hospital, 24 Fukang Road, Nankai, Tianjin 300192, P.R. China

E-mail: kachydeng@126.com

Key words: chimeric antigen receptor-T cells, murine, humanized, central nervous system leukemia, cytokine release syndrome
In Nalm-6 BALB/c mice, the median survival time of mice in the murine CD19 CAR-T group was 35 days, while it was 43 days in the humanized CD19 CAR-T group. In conclusion, humanized CD19 CAR-T cell therapy had a better curative effect than that of murine CD19 CAR-T therapy, and may be used as a salvage treatment for recurrent B-ALL after treatment with murine CD19 CAR-T therapy.

\section{Introduction}

For patients with acute lymphoblastic leukemia (ALL), the accompanying risk of central nervous system leukemia (CNSL) is more severe compared with that of patients with acute myeloid leukemia $(1,2)$. CNSL is one of the primary causes of relapse in patients with acute leukemia and is associated with a less favorable prognosis (3). Initially, patients may achieve CNSL remission in the short term using established treatments, such as chemotherapy, intrathecal chemotherapy and radiation therapy (4).

The second generation of CD19-directed chimeric antigen receptors $(\mathrm{CAR})$ results in a more pronounced activation and expansion of $\mathrm{T}$ cells to eliminate malignant $\mathrm{B}$ cells in vitro and in vivo (5). The use of anti-CD19 chimeric antigen receptor modified $\mathrm{T}$ (CAR-T) cells has achieved 70-88\% $\mathrm{CR}$ and $\mathrm{CRi}$ in recurrent or refractory (R/R) B-ALL $(6,7)$. Although for cases of R/R B-cell ALL, CD19 CAR-T therapy induces rapid and effective responses, it is associated with acute toxicity, including cytokine-release syndrome (CRS) and CAR-T cell-related encephalopathy syndrome (CRES), which are severe or even fatal (8). CRS is characterized by a high fever, hypotension, hypoxia and/or multiorgan toxicity, whereas CRES is typically characterized by confusion, delirium and cerebral edema (9-12). Therefore, CRES is likely to be the more toxic side effect of CD19 CAR-T therapy in patients with CNSL arising from B-ALL.

At present, targeted CD19 CAR-T therapy for R/R $\mathrm{B}$-cell malignant hematopathy is primarily attributed to the single-chain Fvs (scFvs) in CAR structures, which are derived from murine FMC63 or SJ25C1, which are the clone IDs for monoclonal antibodies targeting CD19 $(6,13,14)$. 
In the majority of patients, immune responses are induced by the invasion of mouse-derived scFv structures to CD19 CAR-T cells following therapy (15). Although the effects are significant in the short term, survival of CAR-T cells in the peripheral blood following treatment for a short amount of time may assist the maintenance of the long term therapeutic effect (15). It has been reported that $93 \%$ of patients with $\mathrm{R} / \mathrm{R}$ ALL exhibit a complete response (CR) to $\mathrm{B}$ cells following CD19 CAR-T cell therapy (7).

In a clinical study, 32 patients with ALL were treated with murine CD19 CAR-T therapy, and 5 patients relapsed; after receiving the second infusion, all of them did not respond to any curative effects after reinfusion of murine CD19 CAR-T cells (16). Immunogenicity may be an important factor affecting the survival of CAR-T cells in vivo and the curative effect of murine CD19 CAR-T therapy when used more than once (6). Therefore, humanized CD19 CAR-T cells have been constructed by isolation of anti-human CD19 scFvs from human DNA libraries and replaced with murine FMC63-derived scFv which possesses similar binding characteristics (17). A previous study has demonstrated that host immune responses can recognize the epitopes of the murine $\mathrm{scFv}$ and render subsequent infusions ineffective (18). Humanized CD19 CAR-T cells exhibit improved effects against tumor cell lines compared with murine CD19 CAR-T cells in vitro (19). Research on humanized CD19 CAR-T cells is being performed at several centers around the world; however, there are relatively few reports of humanized CD19 CAR-T therapy in clinical trials. The aim of the present study was to compare the differences between humanized CD19 and murine CD19 CAR-T cell therapy in recurrent B-ALL.

\section{Materials and methods}

Medical history presentation. History prior to murine CD19 CAR-T therapy: A 62-year-old male patient was admitted to Tianjin First Central Hospital (Tianjin, China) due to leukocytosis in September 2013. After bone marrow puncture and flow cytometry, the patient was diagnosed with B-ALL $\left(\mathrm{BCR} / \mathrm{ABL}^{+}\right)$. The patient received VDCP (vincristine, daunorubicin, cyclophosphamide and dexamethasone) chemotherapy and oral imatinib treatment. Subsequently, the first CR (CR1) was achieved in October 2013. In the following two courses of consolidation chemotherapy with VDCP, leukemia cells were found in the cerebrospinal fluid (CSF). The minimal residual disease and the BCR-ABL in the bone marrow was negative at this time. Therefore, the patient was diagnosed with CNSL and received two courses of high dose methotrexate (MTX) combined with intrathecal chemotherapy. CR2 was achieved in May 2014 and maintained with imatinib therapy for the following 17 months. In September 2016, the patient was readmitted with dizziness and tinnitus and diagnosed as relapsed CNSL. The patient received high dose MTX combined with several courses of intrathecal chemotherapy; however, CR was not achieved again.

Murine CD19 CAR-T therapy as a first-time salvage therapy. According to the examination, $57.51 \%$ of leukemia cells were found in the CSF, the patient's minimal residual disease in the bone marrow was $0.24 \%$ and P210 was $0.81 \%$ in February 2017 .
The patient was enrolled in a clinical trial at the Department of Hematology at Tianjin First Central Hospital (Tianjin, China) for treatment with autologous CAR-T 19 cells expressing murine anti-CD19 scFv and 4-1BB-CD3 $\zeta$ costimulatory-activation domains (ChiCTR-ONN-16009862) following relapsed CNSL. Lymphodepleting chemotherapy with fludarabine $\left(30 \mathrm{mg} / \mathrm{m}^{2}\right)$ and cyclophosphamide $\left(400 \mathrm{mg} / \mathrm{m}^{2}\right.$ ) was administered daily between 4 and 2 days prior to initiation of the trial therapy. The patient received four courses of intrathecal chemotherapy to reduce the number of leukemia cells in the CSF from 57.51 to $5.26 \%$ prior to infusion of CAR-T 19 cells. Autologous murine CAR-T 19 cells were infused $\left(1 \times 10^{6}\right.$ cells $/ \mathrm{kg}$ on day 1 and $6 \times 10^{6}$ cells $/ \mathrm{kg}$ on day 2). The transduction efficiency of CD19 CAR and amplification of CD19 CAR-T cells were analyzed by flow cytometry (FCM) (data not shown).

Humanized CD19 CAR-T therapy as a second-time salvage therapy. The patient maintained a stable condition for the following 15 months after treatment with murine CD19 CAR-T therapy combined with oral imatinib. In May 2018, the dizziness, tinnitus and deafness progressed, and the patient was diagnosed with relapsed CNSL again. The patient received high dose MTX combined with several courses of intrathecal chemotherapy as salvage therapy for 6 months. In November 2018, 79.28\% leukemia cells were found in the CSF, while the patient's minimal residual disease in the bone marrow was $3.58 \%$ and $\mathrm{P} 210$ was $32.04 \%$. The patient was given six courses of intrathecal chemotherapy, after which the patient was enrolled in a clinical trial for treatment with autologous CAR-T 19 cells expressing humanized anti-CD19 $\mathrm{ScFv}$ and 4-1BB-CD3 $\zeta$ costimulatory-activation domains (ChiCTR1800019622) as second-time relapsed CNSL. The patient received lymphodepleting chemotherapy in the same manner as the first time. After six courses of intrathecal chemotherapy, the quantity of leukemia cells in the CSF was reduced to $10.25 \%$ prior to CAR-T 19 cell infusion. Autologous CAR-T 19 cells were infused $\left(5 \times 10^{5}\right.$ cells $/ \mathrm{kg}$ on day 1 and $5 \times 10^{5}$ cells/ $\mathrm{kg}$ on day 2 ). Since the patient began to exhibit pyrexia $24 \mathrm{~h}$ after the first infusion, the total number of autologous humanized CAR-T 19 cells was reduced to $1 \times 10^{6}$ cells $/ \mathrm{kg}$ compared with $7 \times 10^{6}$ cells $/ \mathrm{kg}$ in the murine CD19 CAR-T therapy. The transduction efficiency of CD19 CAR and amplification of CD19 CAR-T cells were analyzed by FCM (data not shown).

Isolation of peripheral blood mononuclear cells (PBMCs) and transduction of $T$ cells with murine and humanized CD19 CAR. PBMCs were isolated by Ficoll density gradient centrifugation at $400 \mathrm{xg}$ at $37^{\circ} \mathrm{C}$ for $20 \mathrm{~min} . \mathrm{CD}^{+} \mathrm{T}$ cells were selected from the PBMCs by MACS using CD3 microbeads (Miltenyi Biotec, Inc.). $\mathrm{CD}^{+} \mathrm{T}$ cells were stimulated by anti-CD3/anti-CD28 mAb-coated Human T-Expander beads (cat. no. 11141D; Thermo Fisher Scientific, Inc.) and cultured in T-cell medium X-Vivo 15 (Lonza Group, Ltd.) supplemented with $250 \mathrm{IU} / \mathrm{ml}$ IL-2 (Proluekin; Novartis International AG). After the 4th day of culturing, at which point the $\mathrm{CD} 19^{+}$leukaemia cells could not be detected in the culture by FCM (BD Biosciences), T cells $\left(3 \times 10^{6}\right)$ were transduced with a lentiviral vector encoding CD19-CAR constructs [10 $\mu \mathrm{g}$; lenti-CD19-2rd-CAR (murine/humanized); 
Shanghai Genbase Biotechnology Co., Ltd.] and cultured in medium containing recombinant human IL-2 $(250 \mathrm{U} / \mathrm{ml}$; Proleukin; Novartis International AG) at $37^{\circ} \mathrm{C}$ for 12 days. In the lentivirus packaging process, $\sim 2.5-3.5 \mu \mathrm{g}$ lentiviral vector was packaged in $293 \mathrm{~T}$ cells $\left(1 \times 10^{7}\right)$. The multiplicity of infection (MOI) was 10 in murine CAR-T time and 1 in humanized CAR-T time. After 12 days of cultivation, the transduction efficiencies of anti-CD19 CAR (Shanghai Genbase Biotechnology Co., Ltd.) were analyzed by FCM. A total of $2 \times 10^{5}$ cells were added into each centrifuge tube, $100 \mu 1$ biotin-labeled CD19 protein (cat. no. CD9-H8259; ACROBiosystems) was added into each tube, and then incubated at $4^{\circ} \mathrm{C}$ for $1 \mathrm{~h}$. The final concentration of biotin-labeled $\mathrm{CD} 19$ protein was $10 \mu \mathrm{g} / \mathrm{ml}$. After centrifugation at $300 \mathrm{x} \mathrm{g}$ at $37^{\circ} \mathrm{C}$ for $5 \mathrm{~min}$, the supernatant was discarded and the cells were washed with PBS buffer once. A total of $100 \mu$ l diluted FITC-labeled streptavidin (cat. no. 405201; 1:500; BioLegend, Inc.) was added to each tube. After mixing, the tubes were incubated at $4^{\circ} \mathrm{C}$ for $1 \mathrm{~h}$. After centrifugation at $300 \mathrm{x} \mathrm{g}$ at $37^{\circ} \mathrm{C}$ for $5 \mathrm{~min}$, the supernatant was discarded and the cells were washed three times with PBS buffer. A total of $100 \mu 1$ PBS suspension containing cells were added to each tube. All data were acquired using a BD Fortessa flow cytometer (BD Biosciences) and analyzed using FlowJo software (v10.0.7; FlowJo LLC). The CD19 CAR transfection rate was determined according to the instruction of CARTTEST-19 assay kit (Shanghai Genbase Biotechnology Co., Ltd.).

Primary cells and Nalm-6 cells (American Type Culture Collection) were cultured at $37^{\circ} \mathrm{C}$ in a humidified incubator with $4 \% \mathrm{CO}_{2}$ in RPMI-1640 medium (Gibco; Thermo Fisher Scientific, Inc.) supplemented with $10 \%$ FBS (Gibco; Thermo Fisher Scientific, Inc.) and $50 \mathrm{IU} / \mathrm{ml}$ penicillin/streptomycin (Gibco; Thermo Fisher Scientific, Inc.). The human embryonic kidney 293T (Lenti-X-293T) cells (American Type Culture Collection) were maintained at $37^{\circ} \mathrm{C}$ in a humidified incubator with a $4 \% \mathrm{CO}_{2}$ atmosphere in DMEM (Sigma-Aldrich; Merck KGaA) supplemented with $10 \%$ FBS and $50 \mathrm{UI} / \mathrm{ml}$ penicillin/streptomycin.

Murine and humanized CD19 CAR-T cell proliferation. After the CAR-T cells were harvested, the viability of murine and humanized CD19 CAR-T cells co-cultured with Nalm- 6 cells (effect to target proportion was 1:1) in vitro was detected using a Cell Counting Kit-8 (Dojindo Molecular Technologies, Inc.). A total of $100 \mu 1$ cell suspension (5,000 cells/well) in a 96-well plate were cultured for 0,24 and $48 \mathrm{~h}$ in a humidified incubator at $37^{\circ} \mathrm{C}$. Subsequently, $10 \mu \mathrm{l}$ CCK-8 solution was added to each well of the plate, and the plate was incubated for $4 \mathrm{~h}$ at $37^{\circ} \mathrm{C}$. The absorbance was measured at $450 \mathrm{~nm}$ using a microplate reader. Optical density (OD)450 values were achieved using the Synergy H1 Hybrid Reader (BioTek China) after incubation and analyzed with Gen5 software.

In vitro cytotoxicity to Nalm-6 cells and cytokine-release assays. Two groups of CD19 CAR-T cells were co-cultured at $37^{\circ} \mathrm{C}$ with Nalm- 6 cells at a ratio of $4: 1$ for $48 \mathrm{~h}$ in the absence of supplemented cytokines. Cytotoxicity was detected at 0,24 and $48 \mathrm{~h}$ using a lactate dehydrogenase cytotoxicity test kit (cat. no. C0017; Beyotime Institute of Biotechnology) at according to the manufacturer's instructions at $490 \mathrm{~nm}$. All assays were performed in duplicate or triplicate.

The release of TNF- $\alpha$ and IFN- $\gamma$ from cells was detected using ELISA kits (cat. nos. 555268 and 550612, respectively; BD Biosciences). The absorbance value at $450 \mathrm{~nm}$ was detected at $0,12,24$ and $48 \mathrm{~h}$.

Mouse tumor model establishment and experiment. The mouse tumor model was established based on a previous study by Demehri et al (20). The method of euthanasia was carbon dioxide anesthesia. The animals were placed in a 10-liter euthanasia chamber with a carbon dioxide to oxygen ratio of 6:4. After the animals gradually lost consciousness, the chambers were filled with $100 \%$ carbon dioxide, and the flow rate was $21 / \mathrm{min}$ ( $20 \%$ carbon dioxide/min). Carbon dioxide was increased to $100 \%$ and kept for $10 \mathrm{~min}$ to determine the death of animals (21). Six to eight-week old female CAnN.Cg-Foxn1nu/CrlVR (BALB/c) mice weighing 20.31 \pm 1.39 g ( $n=30$; Charles River Laboratories, Inc.) were used in the present study. BALB/c mice were raised in SPF conditions at $20-26^{\circ} \mathrm{C}$, relative humidity $40-70 \%$ and light and dark alternated 10/14 h cycle. They were fed with food and water sterilized by high temperature and high pressure steam. Mice were injected subcutaneously with $5 \times 10^{6}$ Nalm-6 cells transduced with luciferase (AmyJet Scientific). Mice were monitored with bioluminescence imaging (BLI) for disease progression twice a week following intraperitoneal injection with D-luciferin (150 mg/kg) (AmyJet Scientific) $10 \mathrm{~min}$ before scanning. Before imaging, mice were anesthetized via a nose cone with $2 \%$ isoflurane medical oxygen (Zoetis) and maintained under continuous inhalational anesthesia. All mice were sacrificed wwhen the tumor volume exceeded $20 \mathrm{~mm}^{3}$ or when the experimental study has been completed. The mice were divided into three groups. The first group was given no treatment, the second group was given murine CD19 CAR-T, and the third group was given humanized CD19 CAR-T. The survival time of mice was assessed. The release of IFN- $\gamma$ from peripheral blood was detected by ELISA (cat. no. E4593-100; AmyJet Scientific, Inc.). A total of $500 \mu \mathrm{l}$ of blood was collected from the inner canthus of the three groups on days 7 , 14, 21 and 28. Survival time of mice was the time when mice died or were killed for ethical reasons during the experiment.

Statistical analysis. All experiments were performed independently at least three times and data are presented as the mean \pm SD. Statistical analyses were performed using SPSS 18.0 software (SPSS, Inc.). Comparisons between two groups were made using the independent sample t-test. Comparisons among $>2$ groups were made using one-way ANOVA followed by Fisher's LSD post-hoc test. Survival curves were plotted using the Kaplan-Meier method, and the difference in OS survival rates was assessed using the log-rank test. $\mathrm{P}<0.05$ was considered to indicate a statistically significant difference.

\section{Results}

Expansion of anti-CD19-CAR T-cells during therapy. The transduction efficiency of murine CD19 CAR was $25.88 \%$ (Fig. 1A). Peak levels of expansion of CAR-T cells were 

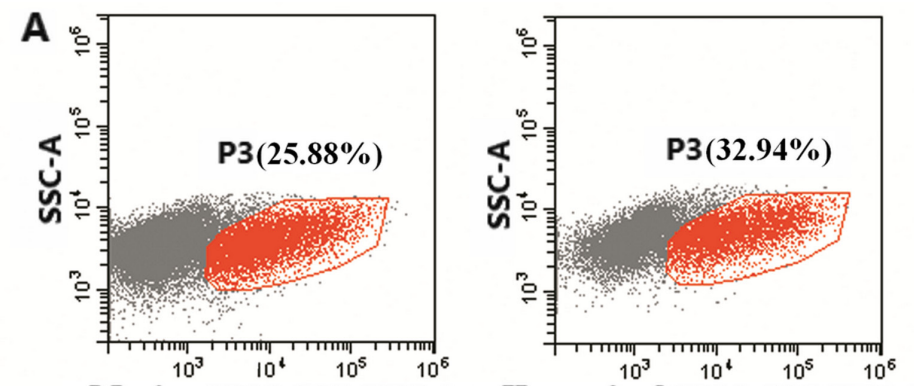

Murine CD19 CAR FITC-A Humanized CD19 CAR FITC-A
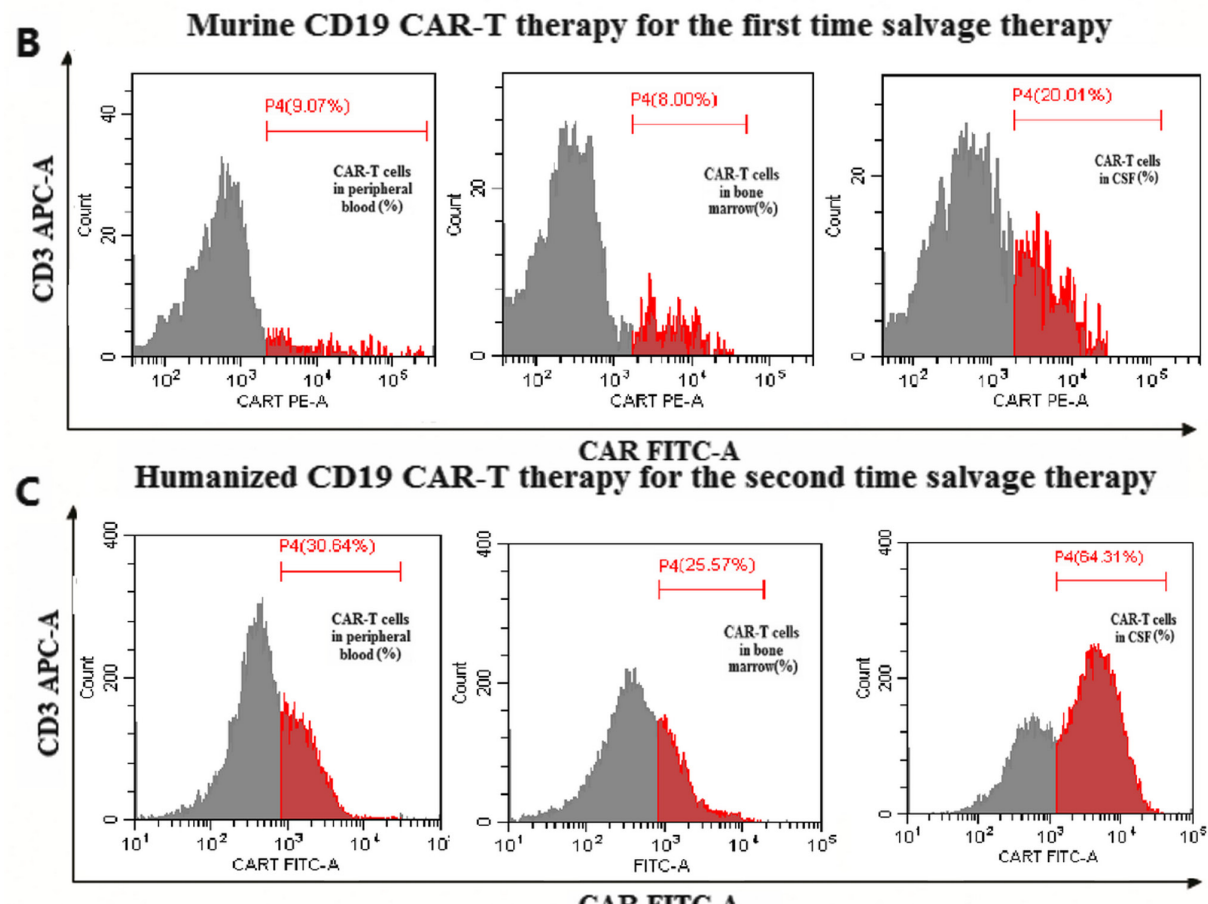

Figure 1. Peak expansion of CAR-T cells in CSF, peripheral blood and bone marrow. The peak expansion of CAR-T cells was observed on day 7. (A) Murine CD19 CAR transduction efficiency was 25.88\%, humanized CD19 CAR transduction efficiency was $32.94 \%$. (B) Peak expression of CD19 CAR-T cells in this murine CD19 CAR-T cell therapy was $20.01 \%$ in CSF, 9.07\% in peripheral blood and $8.00 \%$ in bone marrow. (C) Peak expression of CD19 CAR-T cells in this humanized CD19 CAR-T cell therapy was $64.31 \%$ in CSF, 30.64\% in peripheral blood and $25.57 \%$ in bone marrow. APC, allophycocyanin; CAR-T, CD19 chimeric antigen receptor modified T; CSF, cerebrospinal fluid.

observed on day 7 in CSF accounting for $20.01 \%$ of cells, $9.07 \%$ in the peripheral blood and $8.00 \%$ in bone marrow (Fig. 1B). The time period before all murine CAR-T cells had disappeared was $\sim 14$ days in the CSF, 28 days in the peripheral blood and 42 days in the bone marrow (Fig. 2A-C).

The transduction efficiency of humanized CD19 CAR was $32.94 \%$ (Fig. 1A). Peak expansion of humanized CAR-T cells was observed on day 7 in the CSF accounting for $64.31 \%$ of cells, $30.64 \%$ in the peripheral blood and $25.57 \%$ in the bone marrow (Fig. 1C). The time period before all humanized CAR-T cells had disappeared was $\sim 72$ days in the peripheral blood and 72 days in the bone marrow. Humanized CAR-T 19 cells in the CSF were still present after 42 days of CAR-T 19 cell infusion (Fig. 2A-C).

Adverse events following treatment. The infusions were well tolerated following murine CD19 CAR-T therapy. Notable adverse events were Grade 1 CRS and Grade 1 CRES $(6,9)$. The adverse events manifested as pyrexia $\left(39.7^{\circ} \mathrm{C}\right.$ fever) with chills from days 3-5 following murine CAR-T 19 infusion, accompanied by a headache, dizziness, muscle ache, cognitive impairment, confusion and mild hypotension (Table I). Following murine CD19 CAR-T therapy, the levels of cytokines, including IL-2R, IL-6 and IL-10 were assessed. The highest level of IL-6 in serum was $86.2 \mathrm{pg} / \mathrm{ml}$ on day 7 after infusion (Fig. 3A). IL-2R, IL-8, IL-10 and TNF- $\alpha$ levels peaked on day 7 after infusion of murine CAR-T 19 cells (Fig 3B-E). The levels of cytokines in the CSF were detected by lumbar puncture on day 7 after infusion (Fig. 3). The level of cytokines increased gradually after CAR-T infusion, and the level of cytokines in CSF were higher compared with in peripheral blood from the fourth day after CAR-T infusion (Fig. 3). The patient was given an acetaminophen and diphenhydramine to overcome the adverse events.

The adverse events observed following humanized CAR-T cell therapy were Grade 1 CRS and Grade 2 CRES. Pyrexia $\left(39.7^{\circ} \mathrm{C}\right.$ fever) with chills from days 2-6 after infusion with humanized CD19 CAR-T cells, and headache, dizziness, muscle ache, fatigue, nausea, agitation, confusion and delirium were also experienced by the patient (Table I). The highest level of IL-6 in the serum was $125.3 \mathrm{pg} / \mathrm{ml}$ (on day 7 after infusion (Fig. 3A). The levels of IL-6, IL-8, IL-2R and IL-10 assessed peaked on day 7 after infusion of humanized CD19 CAR-T 
A

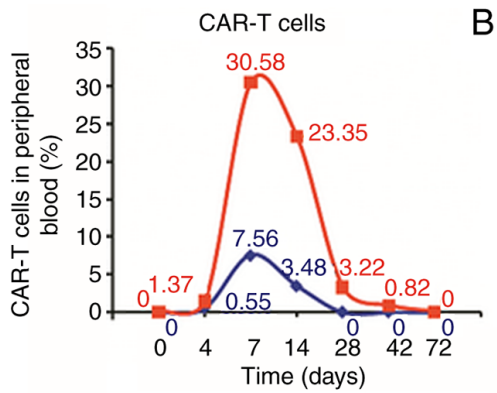

B
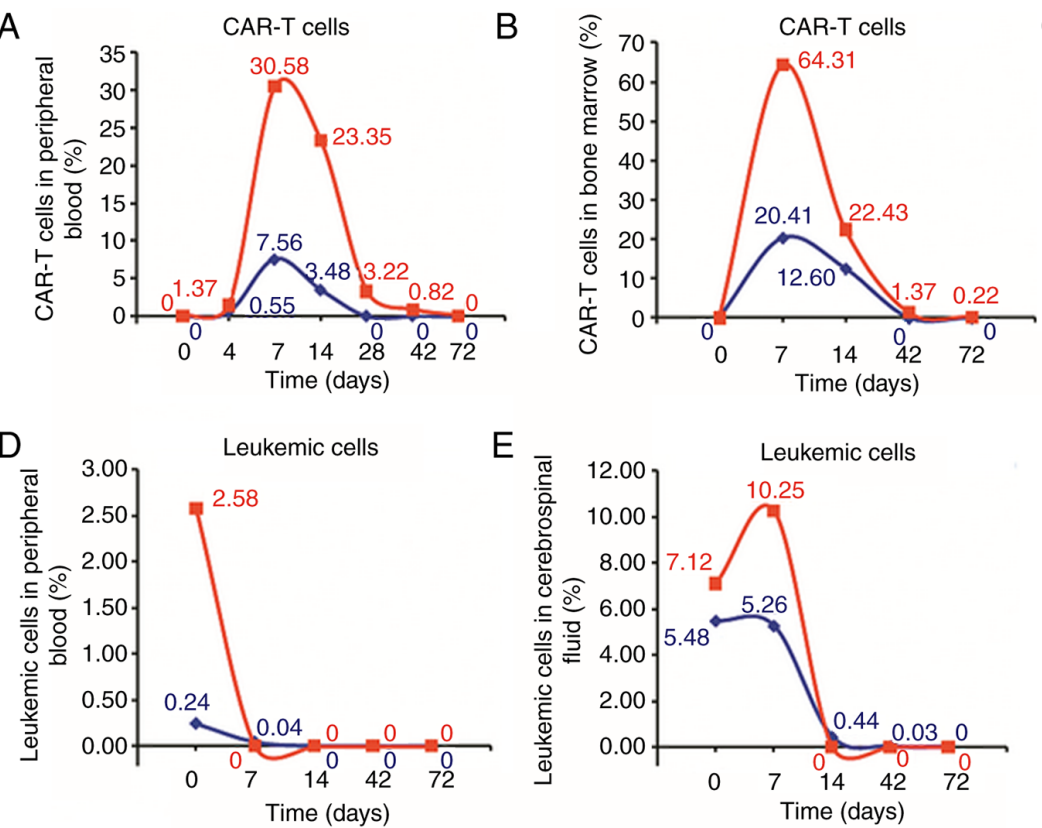

E
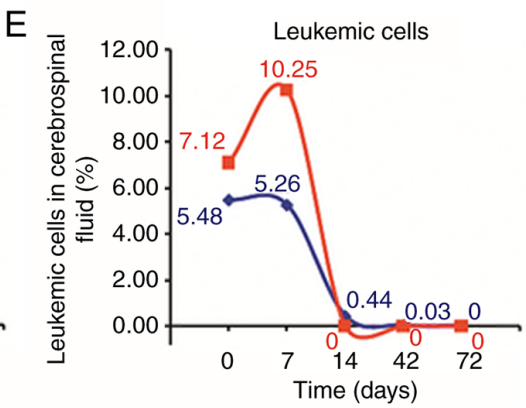

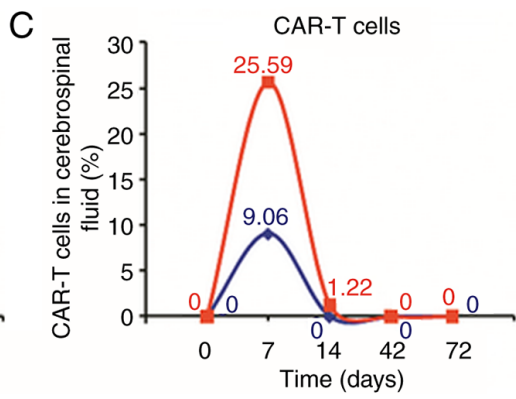

$\leadsto$ Murine CD19 CAR-T therapy

- Humanized CD19 CAR-T therapy

Figure 2. Duration monitoring of CD19 CAR T cells in CSF, peripheral blood and bone marrow. (A-C) The vanishing time of murine CAR-T cells was $\sim 14$ days in CSF, 28 days in peripheral blood and 42 days in bone marrow. The vanishing time of humanized CAR-T cells was 42 days in CSF, 72 days in peripheral blood and 72 days in bone marrow. (D and E) In murine CD19 CAR-T therapy, leukemia cells disappeared at day 72 in CSF and day 14 in peripheral blood. In humanized CD19 CAR-T therapy, leukemia cells disappeared at day 14 in CSF and day 7 in peripheral blood. CAR-T, CD19 chimeric antigen receptor modified T; CSF, cerebrospinal fluid.

and were higher compared with murine CD19 CAR-T infusion. However, the change in TNF- $\alpha$ was not obvious. (Fig. 3B-E). The peak levels of all cytokines in the CSF on day 7 after infusion were lower compared with those in the peripheral blood. In the CSF, the cytokine levels at 7 days in the CSF when treated with humanized CD19 CAR-T therapy were higher compared with those for murine CD19 CAR-T therapy (Fig. 3).

Therapeutic response to CD19-CAR-T cell therapy. The adverse events associated with murine CAR-T cell therapy were relieved after 10 days of infusion and the patient achieved CR after the murine CD19 CAR-T therapy. The disappearance time of leukemic cells from peripheral blood was 14 days after the treatment with murine CD19 CAR-T and 7 days after the treatment with humanized CD19 CAR-T (Fig. 2D). The disappearance time of leukemic cells from CSF was 72 days after the treatment with murine CD19 CAR-T and 14 days after the treatment with humanized CD19 CAR-T (Fig. 2E).

The patient was followed up in the outpatient department and underwent bone marrow biopsy every 2-3 months. He remained in CR condition for the following 18 months after treatment with humanized CD19 CAR-T therapy combined with oral imatinib. Subsequently, the patient relapsed again and declined further treatment.

Effects of murine CD19 CAR-T and humanized CD19 CAR-T on proliferation and cytotoxicity in vitro. The proliferation of humanized CD19 CAR-T cells was higher compared with that of murine CD19 CAR-T cells (24 h, $\mathrm{P}=0.015 ; 48$ h, $\mathrm{P}=0.002$; Fig. 4A). The cytotoxicity of humanized CD19 CAR-T cells was higher compared with the murine CD19 CAR-T cells (24 h, $\mathrm{P}=0.001 ; 48$ h, $\mathrm{P}=0.003$; Fig. 4B).
There was no significant difference between the two groups in terms of the release of TNF- $\alpha$ and IFN- $\gamma$ after $24 \mathrm{~h}$. However, the release of TNF- $\alpha$ and IFN- $\gamma$ following treatment with humanized CD19 CAR-T cells was higher compared with that following treatment with murine CD19 CAR-T cells after $48 \mathrm{~h}$ (TNF- $\alpha, 24$ h, $\mathrm{P}=0.12$ and 48 h, $\mathrm{P}=0.025$; Fig. 4C; IFN- $\gamma$, $24 \mathrm{~h}, \mathrm{P}=0.37$ and $48 \mathrm{~h}, \mathrm{P}=0.011$; Fig. 4D).

Comparison between the murine and humanized CD19 CAR-T therapy in mice. All mice in the PBMCs group died at 7 days after therapy. Tumor fluorescence was barely present in the murine and humanized CD19 CAR-T groups at 7 days after infusion of CAR-T cells, and began to appear 14 days after infusion in the murine CD19 CAR-T group. In the humanized CD19 CAR-T group, fluorescence began to appear at 21 days after infusion. Tumor fluorescence in the murine CD19 CAR-T group rapidly increased in mice treated with murine CD19 CAR-T cells, and the mice began to die 21 days after infusion. Although the tumor fluorescence was also enhanced in the humanized CD19 CAR-T group, the mice survived until 35 days after infusion (Fig. 5A). The median survival time of mice in the murine CD19 CAR-T group was 35 days, and this was 43 days in the humanized CD19 CAR-T group (Fig. 5B). The release of IFN- $\gamma$ in the humanized CD19 CAR-T cells group was increased compared with that in the murine CD19 CAR-T group $(\mathrm{P}<0.001$; Fig. 5C)

\section{Discussion}

The present study investigated the safety and efficacy of humanized CD19 CAR-T therapy as a salvage treatment following murine CD19 CAR-T therapy. The transduction 
Table I. Adverse events in murine and humanized CD19 CAR-T cell therapy.

\begin{tabular}{lll}
\hline Event & Murine CD19 CAR-T & Humanized CD19 CAR-T \\
\hline Fever & Grade 1-CRS & Grade 1-CRS \\
Chills & Grade 1-CRS & Grade 1-CRS \\
Neutropenia & No & No \\
Lymphopenia & No & No \\
Anemia & No & No \\
Thrombocytopenia & No & No \\
Fatigue & Grade 1-CRS & Grade 1-CRS \\
Headache & Grade 1-CRS & Grade 1-CRS \\
Dizziness & Grade 1-CRES & Grade 1-CRES \\
Nausea & No & Grade 2-CRS \\
Vomiting & No & No \\
Somnolence & No & No \\
Agitation & No & Grade 2-CRS \\
Conscious disturbance & Grade 1-CRES & Grade 2-CRES \\
Diminished attention & No & No \\
Language disturbance & No & No \\
Seizures & No & No \\
Ataxia & No & Grade 2-CRS \\
Anorexia & No & No \\
Dyspnea & No & No \\
Delirium & No & Grade 2-CRES \\
Muscle soreness & Grade 1-CRS & Grade 1-CRS \\
Muscle weakness & Grade 1-CRS & Grade 1-CRS \\
Hypotension & Grade 1-CRS & Grade 1-CRS \\
Hypoxemia & No & No \\
Hypoalbuminemia & No & No \\
Transaminase increased & No & No \\
Blood bilirubin increased & No & No \\
Serum creatinine increased & No & No \\
\hline
\end{tabular}

The adverse events that occurred in the humanized CAR-T cell therapy were similar to those that occurred in the murine CAR-T cell therapy. CAR-T, CD19 chimeric antigen receptor modified T; CRES, CAR-T cell-related encephalopathy syndrome; CRS, cytokine-release syndrome.

efficiency, proliferation, efficacy, survival time and the release of cytokines were compared between treatment with murine and humanized CD19 CAR-T cells in vitro and in mice.

CRES is a severe and potentially fatal acute toxicity of CD19 CAR-T therapy for patients with R/R B-cell ALL, despite the promising results of the therapy (22). It has been reported that CAR-T cells are detectable in the CSF of patients who received CD19 CAR-T therapy, indicating severe neurotoxicity $(13,23)$. Another study has demonstrated that the numbers of CAR-T cells in the CSF from patients with severe CRS or CRES were significantly higher compared with those in patients without neurotoxicity (8). The number of CAR-T cells in the peripheral blood also tends to be higher in patients who develop neurotoxicity compared with those who do not (13). In the present study, it was observed that the peak number of CAR-T cells in the CSF was higher compared with that in the peripheral blood or in bone marrow. Therefore, toxicity, and in particular neurotoxicity, may be a severe side effect of the process of CD19 CAR-T therapy for the treatment of patients with CNSL of B-cell ALL.
For patients with significant tumor burdens, particularly patients with ALL, the possibility of serious CRS or CRES is increased $(8,11,24)$. Therefore, reducing the tumor burden may be an important means to prevent the manifestation of the more severe side effects. In the present study, the case of a patient who received several courses of high dose MTX combined with intrathecal chemotherapy to reduce the number of leukemia cells in the CSF before the infusion of CAR-T 19 cells is reported. The notable adverse events were Grade $1 \mathrm{CRS}$ and Grade 2 CRES. Life threatening cerebral edema is the most serious neurotoxic symptoms observed in patients treated with CAR-T cell therapy $(13,25-27)$, since there is a resultant increase to the permeability of the blood-brain barrier and increased levels of cytokines caused by the activation of central nervous system endothelial cells in CD19 CAR-T therapy $(28,29)$. In studies using CAR-T, neurologic adverse events were reported in $40 \%$ of children and young adults with ALL (13\% severe) and $50 \%$ of adults with ALL (50\% severe) $(16,30)$. Therefore, reducing the tumor burden prior to initiation of CD19 CAR-T 

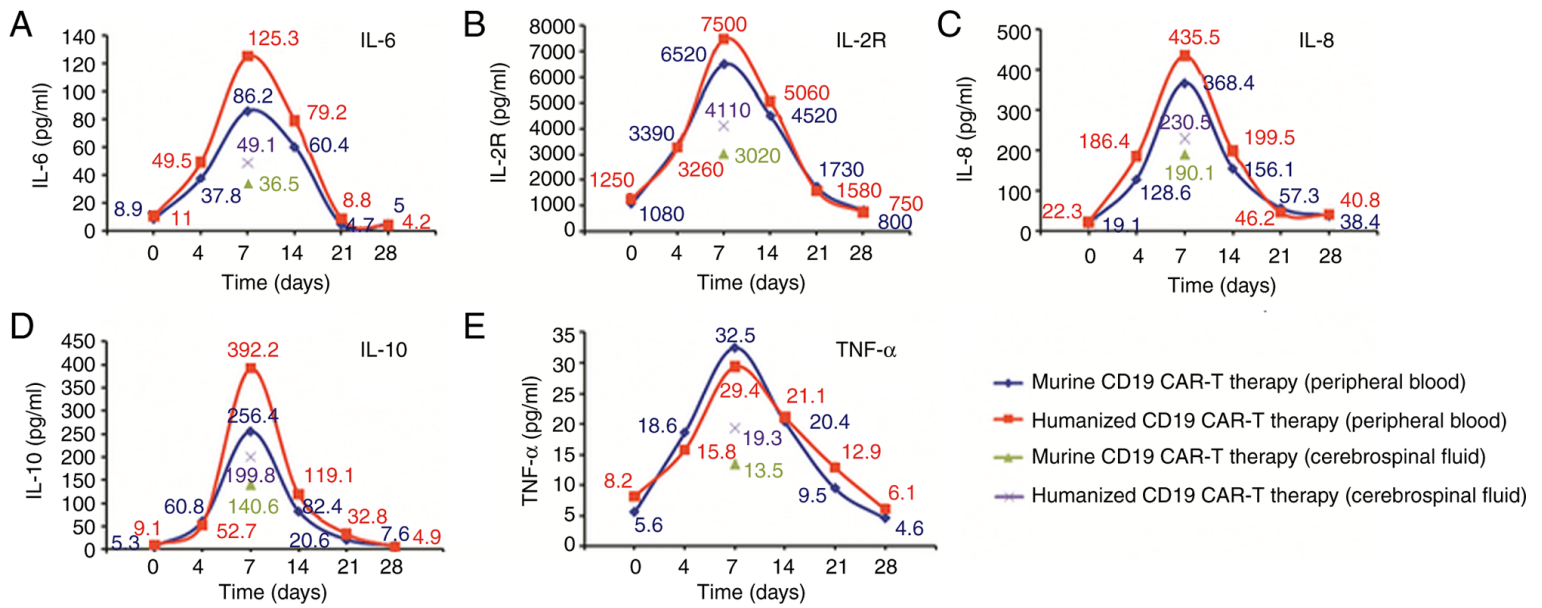

Figure 3. Levels of cytokines including IL-2R, IL-6 and IL-10 and TNF- $\alpha$ were observed during the first and second round of therapy. (A) The highest level of IL-6 in serum on day 7 after infusion was $86.2 \mathrm{pg} / \mathrm{ml}$ in murine CAR-T cell therapy and $125.3 \mathrm{pg} / \mathrm{ml}$ in humanized CAR-T cell therapy. (B) The highest level of IL-2R in serum on day 7 after infusion was $6,520 \mathrm{pg} / \mathrm{ml}$ in murine CAR-T cell therapy and 7,500 pg/ml in humanized CAR-T cell therapy. (C) The highest level of IL-8 in serum on day 7 after infusion was $368.4 \mathrm{pg} / \mathrm{ml}$ in murine CAR-T cell therapy and $435.5 \mathrm{pg} / \mathrm{ml}$ in humanized CAR-T cell therapy. (D) The highest level of IL-10 in serum on day 7 after infusion was $256.4 \mathrm{pg} / \mathrm{ml}$ in murine CAR-T cell therapy and $392.2 \mathrm{pg} / \mathrm{ml}$ in humanized CAR-T cell therapy. (E) The highest level of TNF- $\alpha$ in serum on day 7 after infusion was $29.4 \mathrm{pg} / \mathrm{ml}$ in murine CAR-T cell therapy and $32.5 \mathrm{pg} / \mathrm{ml}$ in humanized CAR-T cell therapy. CAR-T, CD19 chimeric antigen receptor modified T; CSF, cerebrospinal fluid; TNF, tumor necrosis factor.

A
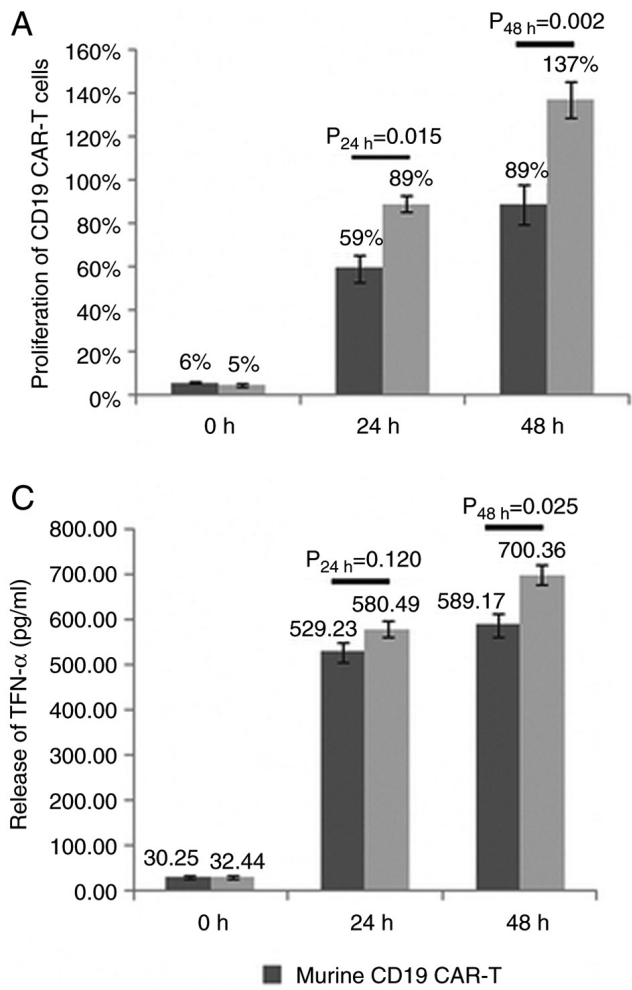

B

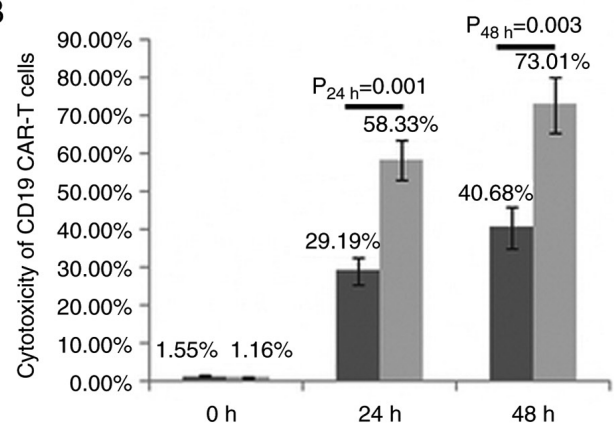

D

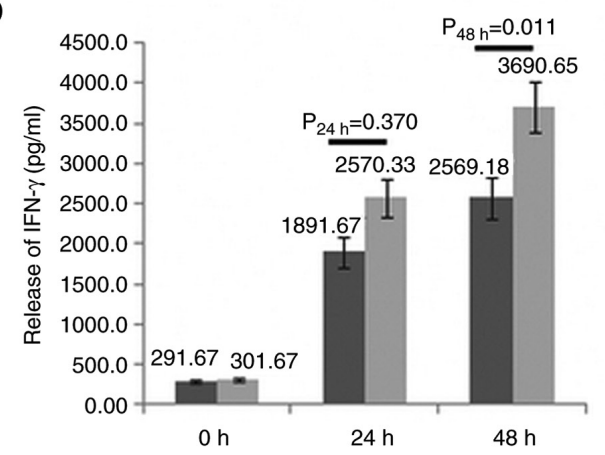

Humanized CD19 CAR-T

Figure 4. Murine and humanized CD19 CAR-T cell transduction efficiency and proliferation. (A) Proliferation of humanized CD19 CAR-T cells was higher than that of murine CD19 CAR-T cells $\left(\mathrm{P}_{24 h}=0.015 ; \mathrm{P}_{48 h}=0.002\right)$. (B) Cytotoxicity of humanized CD19 CAR-T cells was higher than that of murine CD19 CAR-T cells $\left(\mathrm{P}_{24 \mathrm{~h}}=0.001 ; \mathrm{P}_{48 \mathrm{~h}}=0.003\right)$. There was no difference between the two groups at $24 \mathrm{~h}$ in terms of the release of (C) TNF- $\alpha$ and (D) IFN- $\gamma$. However, the release of TNF- $\alpha$ and IFN- $\gamma$ in the humanized CD19 CAR-T cell group was increased compared with that in the murine CD19 CAR-T cell group at $48 \mathrm{~h}$ ( TNF- $\alpha, \mathrm{P}_{24 \mathrm{~h}}=0.120$ and $\mathrm{P}_{48 \mathrm{~h}}=0.025 ; \mathrm{IFN}-\gamma, \mathrm{P}_{24 \mathrm{~h}}=0.370$ and $\mathrm{P}_{48 \mathrm{~h}}=0.011$ ). CAR-T, CD19 chimeric antigen receptor modified $\mathrm{T}$.

therapy may be an effective method for the prevention of life-threatening cerebral edema. For the patient reported on in the present study, there was no cerebral edema reported for treatment with CD19 CAR-T therapy due to the several courses of high dose MTX combined with intrathecal chemotherapy prior to administration of CD19 CAR-T therapy.

Although the most notable therapeutic effect is observed following application of CD19 CAR-T therapy for R/R B 

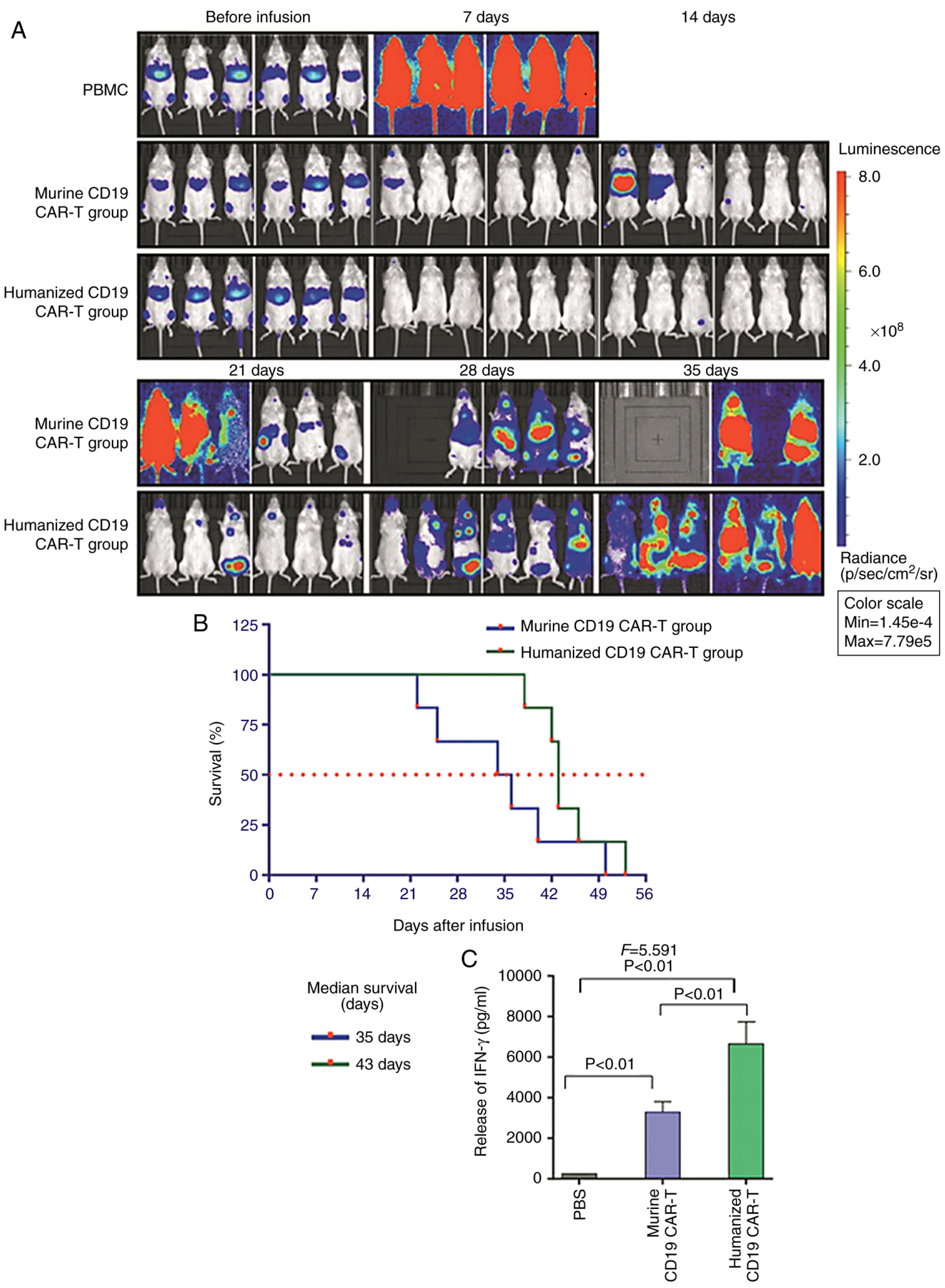

Figure 5. Curative effects of murine and humanized CD19 CAR-T cells in a mouse tumor model. (A) Tumor fluorescence almost disappeared in the murine and humanized CAR-T groups at 7 days after infusion. The tumor fluorescence disappeared 7 days after CA19 CAR-T infusion. The tumor fluorescence began to reappear at 14 days after infusion in the murine CAR-T group, while it began to reappear at 21 days after infusion in the humanized CAR-T group. The mice in the murine CAR-T group died quickly after 21 days of infusion, whereas the mice in the humanized CAR-T group were alive at 35 days after infusion. (B) Median survival of mice in the murine CAR-T group was 35 days compared with 43 days for mice in the humanized CAR-T group. (C) Release of IFN- $\gamma$ in the humanized CAR-T group was higher than that in the murine CAR-T group (ANOVA F=5.591, P<0.01). CAR-T, CD19 chimeric antigen receptor modified T; PBS, blank control group.

cell malignancies, the remission achieved by this therapy is a reflection of its specificity, potency and the persistence of CAR-T cells $(26,31)$. Human leukocyte antigen restricts the T-cell-mediated immune response to epitopes derived from the murine scFv, which may affect the survival time of CAR-T cells in vivo (32). It has been reported that patients treated with murine CD19 CAR-T therapy develop an immune response specific to the murine scFv, 
and thus exhibit subsequent failure to respond to murine CD19 CAR-T therapy (15). Taking advantage of humanized scFvs may reduce the immunogenicity of murine CD19 CAR-T cells and improve the longevity of CAR-T cells in patients (17,33-35). In the present study, humanized CD19 CAR-T cells exhibited higher efficiency towards tumor cell lines in vitro and in mice compared with murine CD19 CAR-T cells. Additionally, the transduction efficiency, proliferation, cytotoxicity to Nalm-6 cells and cytokine-release of humanized CAR-T cells were higher compared with those of murine CAR-T cells in vitro and in mice. Based on the aforementioned experimental results, the number of CD19 CAR-T cells infused in the second therapy was reduced. The total number of autologous humanized CAR-T 19 cells infused was $1 \times 10^{6}$ cells $/ \mathrm{kg}$ in the second therapy compared with $7 \times 10^{6}$ cells/kg in the murine CD19 CAR-T therapy. The second therapy had a complete curative effect and the severity of side effects was the same as for the first therapy. Another promising result observed in the present study was that the survival times of humanized CAR-T 19 cells in the CSF, peripheral blood and bone marrow were longer compared with those of murine CAR-T 19 cells. At this point, the patient remained in remission for $>9$ months after treatment with humanized CD19 CAR-T therapy. Longer remission was achieved and the risk of recurrence was reduced with the humanized CD19 CAR-T therapy. After successfully treating the patient and following recurrence, the patient was entered into another clinical trial for CD19 CAR-T therapy (ChiCTR1800019622). To establish a salvage therapy based on humanized CD19 CAR-T cell therapy for treatment of $\mathrm{R} / \mathrm{R} \mathrm{B}$-cell hematologic malignancy to prolong survival, including patients who failed to respond or relapsed when treated with murine CD19 CAR-T therapy. The advantage of humanized CD19 CAR-T over murine CD19 CAR-T is due to a different humanized $s c F v$ sequence, the reversed order of heavy chain and light chain, and/or incorporation of the selective short peptide, which might have contributed to a change in the three-dimensional configuration of the antigen-recognizing domain of CAR (18).

In conclusion, the present study investigated the efficacy of humanized CD19 CAR-T as a salvage therapy for recurrent CNS B-ALL following murine CD19 CAR-T therapy. The prevention of life-threatening neurotoxicity, such as cerebral edema, following treatment with humanized CD19 CAR-T therapy may be achieved by reducing the initial tumor burden prior to initiation of treatment.

\section{Acknowledgements}

Not applicable.

\section{Funding}

No funding was received.

\section{Availability of data and materials}

The datasets used and/or analyzed during the current study are available from the corresponding author on reasonable request.

\section{Authors' contributions}

QD conceived and designed the study. XL, ZXY and NM performed the experiments. MJL and JM contributed to acquisition of data. HBZ and JW contributed to analysis and interpretation of data. XL wrote, reviewed and revised the manuscript. XL and QD confirmed the authenticity of all the raw data. All authors read and approved the manuscript and agree to be accountable for all aspects of the research in ensuring that the accuracy or integrity of any part of the work are appropriately investigated and resolved.

\section{Ethics approval and consent to participate}

The present study was approved by the Ethics Committee of Tianjin First Central Hospital (Tianjin, China), and written informed consent was obtained from the enrolled patient. Additionally, the patient data were treated in accordance with the local privacy regulations. All animal procedures were approved by the institutional animal and care use committee of Tianjin First Central Hospital (Tianjin, China).

\section{Patient consent for publication}

The patient provided written informed consent.

\section{Competing interests}

The authors declare that they have no competing interests.

\section{References}

1. Rozovski U, Ohanian M, Ravandi F, Garcia-Manero G, Faderl S, Pierce S, Cortes J and Estrov Z: Incidence of and risk factors for involvement of the central nervous system in acute myeloid leukemia. Leuk Lymphoma 56: 1392-1397, 2015.

2. Aoki J, Ishiyama K, Taniguchi S, Fukuda T, Ohashi K, Ogawa H, Kanamori H, Eto T, Iwato K, Sakamaki H, et al: Outcome of allogeneic hematopoietic stem cell transplantation for acute myeloid leukemia patients with central nervous system involvement. Biol Blood Marrow Transplant 20: 2029-2033, 2014.

3. Krishnan S, Wade R, Moorman AV, Mitchell C, Kinsey SE, Eden TO, Parker C, Vora A, Richards S and Saha V: Temporal changes in the incidence and pattern of central nervous system relapses in children with acute lymphoblastic leukaemia treated on four consecutive Medical Research Council trials, 1985-2001. Leukemia 24: 450-459, 2010.

4. Jin MW, Xu SM and An Q: Central nervous disease in pediatric patients during acute lymphoblastic leukemia (ALL): A review. Eur Rev Med Pharmacol Sci 22: 6015-6019, 2018.

5. Irving BA and Weiss A: The cytoplasmic domain of the T cell receptor zeta chain is sufficient to couple to receptor-associated signal transduction pathways. Cell 64: 891-901, 1991.

6. Davila ML, Riviere I, Wang X, Bartido S, Park J, Curran K, Chung SS, Stefanski J, Borquez-Ojeda O, Olszewska M, et al: Efficacy and toxicity management of $19-28 z$ CAR T cell therapy in B cell acute lymphoblastic leukemia. Sci Transl Med 6: 224ra25, 2014.

7. Lee DW, Kochenderfer JN, Stetler-Stevenson M, Cui YK, Delbrook C, Feldman SA, Fry TJ, Orentas R, Sabatino M, Shah NN, et al: T cells expressing CD19 chimeric antigen receptors for acute lymphoblastic leukaemia in children and young adults: A phase 1 dose-escalation trial. Lancet 385 : $517-528,2015$.

8. Neelapu SS, Tummala S, Kebriaei P, Wierda W, Gutierrez C, Locke FL, Komanduri KV, Lin Y, Jain N, Daver N, et al: Chimeric antigen receptor T-cell therapy - assessment and management of toxicities. Nat Rev Clin Oncol 15: 47-62, 2018. 
9. Lee DW, Gardner R, Porter DL, Louis CU, Ahmed N, Jensen M, Grupp SA and Mackall CL: Current concepts in the diagnosis and management of cytokine release syndrome. Blood 124: 188-195, 2014. Erratum in: Blood 128: 1533, 2016.

10. Brudno JN and Kochenderfer JN: Toxicities of chimeric antigen receptor T cells: Recognition and management. Blood 127: 3321-3330, 2016.

11. Maude SL, Barrett D, Teachey DT and Grupp SA: Managing cytokine release syndrome associated with novel $\mathrm{T}$ cell-engaging therapies. Cancer J 20: 119-122, 2014.

12. Hu Y, Sun J, Wu Z, Yu J, Cui Q, Pu C, Liang B, Luo Y, Shi J, Jin A, et al: Predominant cerebral cytokine release syndrome in CD19-directed chimeric antigen receptor-modified $\mathrm{T}$ cell therapy. J Hematol Oncol 9: 70, 2016.

13. Lamers CH, Willemsen R, van Elzakker P van Steenbergen-Langeveld S, Broertjes M, Oosterwijk-Wakka J, Oosterwijk E, Sleijfer S, Debets R and Gratama JW: Immune responses to transgene and retroviral vector in patients treated with ex vivo-engineered T cells. Blood 117: 72-82, 2011.

14. Grupp SA, Kalos M, Barrett D, Aplenc R, Porter DL, Rheingold SR, Teachey DT, Chew A, Hauck B, Wright JF, et al: Chimeric antigen receptor-modified T cells for acute lymphoid leukemia. N Engl J Med 368: 1509-1518, 2013.

15. Mulazzani M, Fräßle SP, von Mücke-Heim I, Langer S, Zhou X, Ishikawa-Ankerhold H, Leube J,Zhang W, Dötsch S, Svec M, et al: Long-term in vivo microscopy of CAR T cell dynamics during eradication of CNS lymphoma in mice. Proc Natl Acad Sci USA 116: 24275-24284, 2019.

16. Turtle CJ, Hanafi LA, Berger C, Gooley TA, Cherian S, Hudecek M, Sommermeyer D, Melville K, Pender B, Budiarto TM, et al: CD19 CAR-T cells of defined CD4 ${ }^{+}: \mathrm{CD} 8^{+}$ composition in adult B cell ALL patients. J Clin Invest 126 $2123-2138,2016$

17. Sommermeyer D, Hill T, Shamah SM, Salter AI, Chen Y, Mohler KM and Riddell SR: Fully human CD19-specific chimeric antigen receptors for T-cell therapy. Leukemia 31: 2191-2199, 2017

18. Song DG, Ye Q, Poussin M, Liu L, Figini M and Powell DJ Jr: A fully human chimeric antigen receptor with potent activity against cancer cells but reduced risk for off-tumor toxicity. Oncotarget 6: 21533-21546, 2015.

19. Zhao Y, Liu Z, Wang X, Wu H, Zhang J, Yang J, Zhang F, Liu L, Long J, Lu P, et al: Treatment with humanized selective CD19CAR-T cells shows efficacy in highly treated B-ALL patients who have relapsed after receiving murine-based CD19CAR-T therapies. Clin Cancer Res 25: 5595-5607, 2019.

20. Demehri S, Corbin A, Loriaux M, Druker BJ and Deininger MW: Establishment of a murine model of aggressive systemic mastocytosis/mast cell leukemia. Exp Hematol 34: 284-288, 2006.

21. Tsukahara T, Ohmine K, Yamamoto C, Uchibori R, Ido H, Teruya T, Urabe M, Mizukami H, Kume A, Nakamura M, et al: CD19 target-engineered T-cells accumulate at tumor lesions in human B-cell lymphoma xenograft mouse models. Biochem Biophys Res Commun 438: 84-89, 2013.

22. Liu D and Zhao J: Cytokine release syndrome: Grading, modeling, and new therapy. J Hematol Oncol 11: 121, 2018.

23. Maude SL, Frey N, Shaw PA, Aplenc R, Barrett DM, Bunin NJ, Chew A, Gonzalez VE, Zheng Z, Lacey SF, et al: Chimeric antigen receptor $\mathrm{T}$ cells for sustained remissions in leukemia. N Engl J Med 371: 1507-1517, 2014
24. Kochenderfer JN, Dudley ME, Carpenter RO, Kassim SH, Rose JJ, TelfordWG,HakimFT,HalversonDC,FowlerDH,Hardy NM,etal: Donor-derived CD19-targeted $\mathrm{T}$ cells cause regression of malignancy persisting after allogeneic hematopoietic stem cell transplantation. Blood 122: 4129-4139, 2013.

25. Turtle CJ, Hay KA, Hanafi LA, Li D, Cherian S, Chen X, Wood B, Lozanski A, Byrd JC, Heimfeld S, et al: Durable molecular remissions in chronic lymphocytic leukemia treated with CD19-specific chimeric antigen receptor modified $\mathrm{T}$ cells after failure of ibrutinib. J Clin Oncol 35: 3010-3020, 2017.

26. Johnson LA and June CH: Driving gene-engineered T cell immunotherapy of cancer. Cell Res 27: 38-58, 2017.

27. Lowe KL, Mackall CL, Norry E, Amado R, Jakobsen BK and Binder G: Fludarabine and neurotoxicity in engineered T-cell therapy. Gene Ther 25: 176-191, 2018

28. Jain MD, Bachmeier CA, Phuoc VH and Chavez JC: Axicabtagene ciloleucel (KTE-C19), an anti CD19 CAR T therapy for the treatment of relapsed/refractory aggressive B cell non Hodgkin's lymphoma. Ther Clin Risk Manag 14: 1007-1017, 2018.

29. Gust J, Hay KA, Hanafi LA, Li D, Myerson D, Gonzalez-Cuyar LF, Yeung C, Liles WC, Wurfel M, Lopez JA, et al: Endothelial activation and bloodbrain barrier disruption in neurotoxicity after adoptive immunotherapy with CD19 CAR T cells. Cancer Discov 7: 1404-1419, 2017.

30. Gardner RA, Finney O, Annesley C, Brakke H, Summers C, Leger K, Bleakley M,Brown C, Mgebroff S, Kelly-Spratt KS, et al: Intent-to-treat leukemia remission by CD19 CAR T cells of defined formulation and dose in children and young adults. Blood 129: 3322-3331, 2017

31. Brentjens RJ, Rivière I, Park JH, Davila ML, Wang X, Stefanski J, Taylor C, Yeh R, Bartido S, Borquez-Ojeda O, et al: Safety and persistence of adoptively transferred autologous CD19-targeted T cells in patients with relapsed or chemotherapy refractory B-cell leukemias. Blood 118: 4817-4828, 2011.

32. Mirzaei HR, Pourghadamyari H, Rahmati M, Mohammadi A, Nahand JS, Rezaei A, Mirzaei H and Hadjati J: Gene-knocked out chimeric antigen receptor (CAR) T cells: Tuning up for the next generation cancer immunotherapy. Cancer Lett 423: 95-104, 2018.

33. Johnson LA, Scholler J, Ohkuri T, Kosaka A, Patel PR, McGettigan SE, Nace AK, Dentchev T, Thekkat P, Loew A, et al: Rational development and characterization of humanized anti-EGFR variant III chimeric antigen receptor $\mathrm{T}$ cells for glioblastoma. Sci Transl Med 7: 275ra22, 2015.

34. Alonso-Camino V, Sánchez-Martín D, Compte M, Nuñez-Prado N, Diaz RM, Vile R and Alvarez-Vallina L: CARbodies: Human antibodies against cell surface tumor antigens selected from repertoires displayed on $\mathrm{T}$ cell chimeric antigen receptors. Mol Ther Nucleic Acids 2: e93, 2013.

35. Zhao Y, Wang QJ, Yang S, Kochenderfer JN, Zheng Z, Zhong X, Sadelain M, Eshhar Z, Rosenberg SA and Morgan RA: A herceptin-based chimeric antigen receptor with modified signaling domains leads to enhanced survival of transduced $\mathrm{T}$ lymphocytes and antitumor activity. J Immunol 183: 5563-5574, 2009. International (CC BY-NC-ND 4.0) License. 